\section{Cureus}

\title{
Pauci-immune Glomerulonephritis in Systemic Lupus Erythematosus (SLE)
}

\author{
Jason Liebowitz $^{1}$, Derek Fine ${ }^{2}$, Philip Seo ${ }^{3}$, Michelle Petri ${ }^{3}$, Kirthi Machireddy ${ }^{4}$, Uzma \\ Haque $^{3}$, Rebecca Manno ${ }^{3}$, Homa Timlin ${ }^{5}$ \\ 1. Medicine, The Johns Hopkins University School of Medicine, Baltimore, USA 2. Nephrology, Johns \\ Hopkins Bayview Medical Center, Baltimore, USA 3. Rheumatology, The Johns Hopkins University \\ School of Medicine, Baltimore, USA 4. University of the Sciences, Philadelphia, USA 5. Medicine, The \\ Johns Hopkins University School of Medicine, Essex, USA
}

$\square$ Corresponding author: Homa Timlin, htimlin1@jhmi.edu

Disclosures can be found in Additional Information at the end of the article

\section{Abstract}

Glomerulonephritis (GN) in lupus is generally an immune complex glomerulonephritis from the deposition of immunoglobulin and complements. Pauci-immune GN is the most common cause of rapidly progressive GN and is frequently associated with an anti-nuclear cytoplasmic antibody (ANCA). We report a patient with a history of systemic lupus erythematosus who presented with worsening proteinuria and was subsequently diagnosed with pauci-immune GN on renal biopsy, in the absence of ANCA.

Categories: Allergy/Immunology, Nephrology, Rheumatology

Keywords: pauci-immune glomerulonephritis, lupus

\section{Introduction}

Renal involvement in systemic lupus erythematosus (SLE) is usually an immune complex glomerulonephritis. Pauci-immune necrotizing and crescentic glomerulonephritis (NCGN) refers to extensive glomerular inflammation with few or no immune deposits that may result in a rapid decline in renal function. Pauci-immune glomerulonephritis is frequently associated with an anti-nuclear cytoplasmic antibody (ANCA) [1]. An overlap syndrome of lupus and ANCA-associated vasculitis is rare, with an estimated prevalence of $2 \%$ [2]. Pauci-immune glomerulonephritis (GN) in lupus was first described in 1983 in four patients who had minimal serologic activity [3]. Subsequent reports of pauci-immune glomerulonephritis have included patients with serologic activity of lupus, positive anti-cardiolipin antibody, and evidence of thrombotic microangiopathy on biopsy, with the interval between diagnosis of SLE and diagnosis of pauci-immune glomerulonephritis ranging from two months to 12 years [4]. Although $15 \%$ to $20 \%$ of lupus patients have circulating ANCA, these reports included patients who were ANCA-negative [5]. ANCAs appear to influence the histological pattern of lupus nephritis and are associated with worse baseline renal function and more active lupus serology [6]. We present a patient with SLE who developed proteinuria 13 years after her original diagnosis and underwent renal biopsies that revealed pauci-immune necrotizing and crescentic GN in the setting of negative ANCAs.

\section{Case Presentation}

A woman in her late 30s, with a history of SLE characterized by positive anti-nuclear antibodies, anti-Smith antibodies (1:160), anti-double-stranded deoxyribonucleic acid (antidsDNA) antibodies (> 1:640), anti-ribonucleoprotein (anti-RNP) antibodies, lupus 
anticoagulant, immunoglobulin $\mathrm{M}$ (IgM) anti-cardiolipin antibodies (27, normal: 0 - $12 \mathrm{U} / \mathrm{ml}$ ), hypocomplementemia (C3 < 40, normal: 90 - $165 \mathrm{mg} / \mathrm{dl}$; C4 < 8, normal: 10 - $40 \mathrm{mg} / \mathrm{dl}$ ), rheumatoid factor of $50 \mathrm{IU} / \mathrm{mL}$ (negative: < 13.9), elevated erythrocyte sedimentation rate (ESR), arthritis, lymphopenia, and thrombocytopenia, presented to our clinic with new proteinuria (spot urine protein/creatinine ratio $1,059 \mathrm{mg} / \mathrm{g}$ ) and a normal creatinine $(0.6 \mathrm{mg} / \mathrm{dl})$. Evaluation for antibodies of perinuclear (p)-ANCA, cytoplasmic (c)-ANCA, and atypical pANCA were all negative. She underwent a renal biopsy that demonstrated focal, mild necrotizing crescentic glomerulonephritis. Glomerular staining showed non-specific 1+ linear staining of the glomerular basement membrane and rare 1+ granular mesangial staining for IgG, IgM, and kappa but with negative staining for immunoglobulin A (IgA), C3, C1q, and lambda. Taken together, this renal biopsy was summarized as a pauci-immune focal necrotizing/extracapillary proliferative glomerulonephritis. The patient was treated with rituximab, mycophenolate mofetil (3g daily), and prednisone but continued to demonstrate proteinuria with $0.73 \mathrm{~g} / 24$ hours, creatinine of $0.7 \mathrm{mg} / \mathrm{dl}$, and no hematuria. She underwent a second renal biopsy (at a time when serologies demonstrated low C3 at $77 \mathrm{mg} / \mathrm{dl}$ and positive anti-dsDNA antibodies), which resembled class II lupus nephritis with limited subcapsular glomerular sample with deposits staining for IgG, IgM, kappa light chain, and C3, along capillary loops and peripheral mesangium with sparse deposits on electron microscopy. Given the concerns for steroid side effects, a change in insurance, and the lack of follow-ups, she was maintained on mycophenolate mofetil, $3 \mathrm{~g}$ daily, and a low dose of prednisone, $5 \mathrm{mg}$, along with lisinopril. However, she continued to experience prolonged morning joint stiffness and her hand MRI demonstrated synovitis. Adding methotrexate led to significant improvement in her arthritis. By 23 months after the initial renal biopsy, she continued to have proteinuria, intermittently low $\mathrm{C} 3$, and positive anti-dsDNA antibodies and underwent a third renal biopsy. This biopsy, similar to the first sample, demonstrated a pauci-immune focal proliferative segmental crescentic/necrotizing lesion in 30\% of the glomeruli. On repeat serologic testing, her c-ANCAs, p-ANCAs, and atypical ANCAs were still negative.

\section{Discussion}

Pauci-immune glomerulonephritis is a type of rapidly progressive glomerulonephritis which is generally associated with ANCA positive vasculitis, whereas SLE nephritis is known to be due to immune complex and complements deposition giving it a "full house" pattern on

immunofluorescence. Pauci-immune glomerulonephritis represents almost $80 \%$ of all cases of rapidly progressive glomerulonephritis and its incidence in the United States per year is about 7-10 cases per million [6]. Rapidly progressive glomerulonephritis (RPGN) is classified as pauciimmune glomerulonephritis (PIGN), immune-mediated glomerulonephritis, and antiglomerular basement membrane (anti-GBM) glomerulonephritis. It is estimated that about $80 \%$ of patients with pauci-immune glomerulonephritis have myeloperoxidase (MPO) and p-ANCA, whereas the rest have anti-proteinase 3 (PR3)-cytoplasmic ANCA antibodies (c-ANCA) [7]. Lightstone et al. compared 32 ANCA-positive biopsies to 222 ANCA negative biopsies from patients with lupus nephritis. The majority (82\%) of ANCA-positive patients had antimyeloperoxidase antibodies. Class IV-S lupus nephritis and glomerular necrosis were more common, and isolated Class V lupus nephritis was less common in the ANCA-positive group. ANCA-positive patients had significantly higher dsDNA titers, and higher serum creatinine at the time of biopsy [6].

\section{Conclusions}

Pauci-immune glomerulonephritis in the background of SLE (with negative ANCA) is an unusual presentation that was seen in our patient. The absence of immune deposits suggests that antibody-independent cell-mediated immunity may mediate pauci-immune GN. Further scientific data is needed to determine the absolute prevalence and pathophysiology of this condition. 


\section{Additional Information \\ Disclosures}

Human subjects: Consent was obtained by all participants in this study. Conflicts of interest: In compliance with the ICMJE uniform disclosure form, all authors declare the following:

Payment/services info: All authors have declared that no financial support was received from any organization for the submitted work. Financial relationships: All authors have declared that they have no financial relationships at present or within the previous three years with any organizations that might have an interest in the submitted work. Other relationships: All authors have declared that there are no other relationships or activities that could appear to have influenced the submitted work.

\section{References}

1. Syed R, Rehman A, Valecha G, El-Sayegh S : Pauci-immune crescentic glomerulonephritis: an ANCA-associated vasculitis. Biomed Res Int. 2015, 2015:402826. 10.1155/2015/402826

2. Jarrot PA, Chiche L, Hervier B, et al.: Systemic lupus erythematosus and antineutrophil cytoplasmic antibody-associated vasculitis overlap syndrome in patients with biopsy-proven glomerulonephritis. Medicine (Baltimore). 2016, 95:e3748. 10.1097/MD.0000000000003748

3. Schwartz M, Roberts J, Lewis E: Necrotizing glomerulitis of systemic lupus erythematosus. Hum Pathol. 1983, 14:158-67. 10.1016/S0046-8177(83)80245-7

4. Charney D, Nassar G, Truong L, Nadasdy T: "Pauci-immune" proliferative and necrotizing glomerulonephritis with thrombotic microangiopathy in patients with systemic lupus erythematosus and lupus-like syndrome . Am J Kidney Dis. 2000, 35:1193-206.

10.1016/S0272-6386(00)70058-8

5. Sen D, Isenberg D: Antineutrophil cytoplasmic autoantibodies in systemic lupus erythematosus. Lupus. 2003, 12:651-58. 10.1191/0961203303lu456rr

6. Turner-Stokes T, Wilson HR, Morreale M, et al.: Positive antineutrophil cytoplasmic antibody serology in patients with lupus nephritis is associated with distinct histopathologic features on renal biopsy. Kidney Int. 2017, 92:1223-31. 10.1016/j.kint.2017.04.029

7. Seo P, Stone J: The antineutrophil cytoplasmic antibody-associated vasculitides. Am J Med. 2004, 117:39-50. 10.1016/j.amjmed.2004.02.030 